\title{
The Influence of Viscous Operator and Wall Boundary Conditions on the Accuracy of the Navier-Stokes Equations
}

Peter Eliasson and Jan Nordström

\section{Linköping University Post Print}

\section{Tweet}

N.B.: When citing this work, cite the original article.

Original Publication:

Peter Eliasson and Jan Nordström, The Influence of Viscous Operator and Wall Boundary Conditions on the Accuracy of the Navier-Stokes Equations, 2013, AIAA Aerospace Sciences - Fluid Sciences Event. http://dx.doi.org/10.2514/6.2013-2956

From the 21st AIAA Computational Fluid Dynamics Conference 24 - 27 June 2013 San Diego, California. 


\title{
The Influence of Viscous Operator and Wall Boundary Conditions on the Accuracy of the Navier-Stokes Equations
}

\author{
Peter Eliasson \\ Swedish Defence Research Agency (FOI), SE-16490, Stockholm, Sweden \\ Jan Nordström \\ Linköping University, Department of Mathematics, SE-581 83, Linköping, Sweden
}

\begin{abstract}
The discretization of the viscous operator in an edge-based flow solver for unstructured grids has been investigated. A compact discretization of the Laplace and thin-layer operators in the viscous terms is used with two different wall boundary conditions. Furthermore, a wide discretization of the same operators is investigated. The resulting numerical operators are all formally second order accurate in space; the wide operator has higher truncation errors. The operators are implemented weakly using a penalty formulation and are shown to be stable for a scalar model problem with given constraints on the penalty coefficients. The different operators are applied to a set of grid convergence test cases for laminar flow in two dimensions up to a large-scale three dimensional turbulent flow problem. The operators converge to the same solutions as the grids are refined with one exception where the wide operator converges to a solution with higher $\mathrm{drag}$. The $2^{\text {nd }}$ compact discretization, being locally more accurate at a wall boundary than the original $1^{\text {st }}$ compact operator, reduces the grid dependency somewhat for most test cases. The wide operator performs very well and leads for most test cases to results with minimum spread between coarsest and finest grids. For one test case though, the wide operator has a negative influence on the steady state convergence.
\end{abstract}

\section{Introduction}

The no-slip wall boundary condition is difficult to implement numerically in a stable and accurate manner. Weak boundary conditions are preferred for node vertex based schemes since stability and accuracy can be shown using Summation-by-Parts (SBP) operators combined with the Simultaneous Approximation Term (SAT) approach ${ }^{1-5}$. This approach has earlier been shown to give satisfactory steady state convergence for viscous flow computations when implemented in a finite volume scheme for unstructured grids ${ }^{6,7}$. That investigation demonstrated how to specify no-slip wall velocity and gave an interval on the penalty strength parameter to ensure stability.

The weak boundary conditions are further explored in this paper by investigating the accuracy and asymptotic convergence as the grid is refined. A compact stencil of the viscous terms is used with the wall boundary conditions from Eliasson et al. ${ }^{6}$. An alternative boundary discretization for the compact operator is explored ${ }^{4}$ which is locally more accurate than the original one. Furthermore, a wider discretization of the viscous terms is also investigated ${ }^{1-3}$. The three approaches are compared and evaluated for a model problem for which stability is shown to hold under conditions on the penalty parameters.

The different numerical schemes are implemented in the Edge flow solver for unstructured grids ${ }^{8-10}$. A compact discretization of the Laplace and thin-layer part of the viscous operator is applied. Two different boundary conditions with different wall fluxes are used resulting in stencils corresponding to those for the model problem. A wide discretization of the viscous operator results in the corresponding wide stencil for the model problem. The three viscous stencils are applied on a number of test cases with focus on how the errors are reduced as the grid is refined. Comparisons are made on simpler laminar cases up to a large scale turbulent test case from the AIAA drag prediction workshop.

In the following sections the weak boundary conditions are introduced and applied to a scalar model problem for which a theoretical analysis is carried out. Then the spectra are analyzed for the linearized Navier-Stokes equations. Following that a description of the flow solver Edge and the wall fluxes for the various viscous operators are given. Then numerical results are presented for the computed test cases followed by a discussion of the results. 


\section{Weak Boundary Conditions}

The flow solver, described below, is of finite volume type with the unknowns in the nodes. All boundary conditions are implemented in weak way which implies that a boundary flux is computed and added to the residual of the unknown boundary quantities which are updated like any other variables in the interior domain. In earlier work ${ }^{6}$ we have shown that weak no-slip wall boundary conditions improve the efficiency with respect to steady state convergence compared to strong conditions where the specified boundary value is injected on the boundary and the boundary quantities are no longer unknowns.

Here we extend our previous work on weak no-slip wall boundary conditions by introducing alternative viscous operators as well as alternative boundary conditions. Our ambition is to enhance the accuracy of flow in the near wall region and, hence, to reduce the differences between flow solutions as the grid is refined.

We consider three different numerical operators of the viscous terms. The first option uses a compact discretization in the interior with a low order accurate boundary treatment ${ }^{6}$. The second operator uses the same compact operator in the interior but has a more accurate boundary treatment. The third variant has a wider noncompact stencil that corresponds to using nodal gradients in the viscous flux calculations, further described below. The weak no-slip condition is applied in exactly the same way for all formulations and all discretizations are formally second order accurate.

Below the different operators are described and analyzed for a scalar model problem.

\section{A. A scalar model problem}

We consider the continuous one-dimensional half plane convection-diffusion model problem

$$
u_{t}+a u_{x}=\varepsilon u_{x x}, \quad u(0, t)=g
$$

where $a>0,0<\varepsilon<<1$. For simplicity we consider the left boundary only, the right boundary requires a similar treatment.

Following the discretization in ${ }^{6,7}$ we use second order accurate operators and end up with the following semidiscrete equations

$$
v_{t}+a P^{-1} Q v=\varepsilon P^{-1} M v-P^{-1}\left(a \tau-\varepsilon \frac{\sigma}{\Delta x}\right) E_{0} v
$$

where it has been assumed that $g=0$. The first derivative $u_{x}$ is approximated such that it satisfies the SBP property, i.e.

$$
u_{x} \approx P^{-1} Q v
$$

where $P=P^{T}>0, Q+Q^{T}=\operatorname{diag}(-1,0, \cdots, 0,1)$. All matrices are given in Appendix. The last terms of Eq. (2) is a generalized penalty term for the convective and viscous parts that force the solution towards the specified boundary value. $\sigma, \tau$ are the penalty strength parameters. In Appendix it is demonstrated that the convective part only ( $\varepsilon=0$ ) is stable provided $\tau \geq 1 / 2$. Below we address the different viscous operators and provide bounds for the viscous penalty strength parameters to obtain stability.

\section{The compact viscous operator 1}

The first viscous operator is currently used in Edge and is the same operator that was first implemented in a weak sense ${ }^{6}$. This operator at the left boundary becomes

$$
P^{-1} M=P^{-1} M_{1}=\frac{1}{\Delta x^{2}}\left[\begin{array}{cccc}
0 & 0 & 0 & \\
1 & -2 & 1 & \\
& 1 & -2 & 1 \\
& & \ddots & \ddots
\end{array}\right] \text {. }
$$


The operator in Eq. (4) is zero order accurate at the boundary which is sufficient for global second order accuracy provided that the solution is point wise bounded ${ }^{11}$. To ensure stability it was shown that $\sigma=\sigma_{1} \geq 2 / 8$ was required and that larger value of $\sigma$ increases the stiffness. The proof of stability is given in Appendix.

\section{The compact viscous operator 2}

To increase the accuracy locally on the boundary an alternative boundary treatment developed by Carpenter et $\mathrm{al}^{4}{ }^{\text {of }}$ the viscous term is proposed. By modifying the boundary flux a different boundary stencil is obtained which is locally more accurate; the intention is to enhance the accuracy of the flow solution locally near the boundary on coarser grids and hence to enhance grid convergence which, in this context, refers to reducing differences between fine and coarse grids. The proposed approach results in the following second derivative operator

$$
P^{-1} M=P^{-1} M_{2}=\frac{1}{\Delta x^{2}}\left[\begin{array}{cccc}
1 & -2 & 1 & \\
1 & -2 & 1 & \\
& 1 & -2 & 1 \\
& & \ddots & \ddots
\end{array}\right]
$$

where the stencils for the second derivative for the boundary and the first interior nodes are the same. This operator is first order accurate on the boundary. To ensure stability it is shown in Appendix that $\sigma=\sigma_{2} \geq 5 / 8$ is required.

\section{The wide viscous operator}

A third variant of the discretization of the viscous term is also considered; the discretization is obtained by applying the central first difference operator $P^{-1} Q$ twice ${ }^{5,7}$. This operator corresponds to what is obtained when averaging nodal gradients in the flux calculation of an Edge based scheme and is hence easily implemented. The resulting viscous operator becomes

$$
P^{-1} M=P^{-1} M_{3}=P^{-1} Q P^{-1} Q=\frac{1}{4 \Delta x^{2}}\left[\begin{array}{ccccc}
2 & -4 & 2 & & \\
2 & -3 & 0 & 1 & \\
1 & 0 & -2 & 0 & 1 \\
& & & \ddots &
\end{array}\right] .
$$

This wide operator is zero order accurate at the first two grid nodes, but again, that is sufficient for global second order accuracy if the scheme is point-wise bounded ${ }^{11}$. The operator has a larger truncation error than the compact one. In Appendix it is shown that stability is obtained provided $\sigma=\sigma_{3} \geq 4 / 8$.

\section{B. System of equations}

We expand our model problem to a system of equations. The model equation is obtained by considering the two dimensional frozen coefficient Navier-Stokes equations. A Fourier-transform is applied tangential to the solid boundary and we consider the least dissipative case, namely the one corresponding to zero frequency $(\omega=0)$. We then neglect the equation for the tangential velocity. The system we obtain and the boundary conditions at the solid boundary are

$$
U_{t}+A U_{x}=\varepsilon B U_{x x}, \quad u(0, t)=0, \quad T_{x}(0, t)=0
$$

where

$$
A=\left[\begin{array}{lll}
\bar{u} & a & 0 \\
a & \bar{u} & b \\
0 & b & \bar{u}
\end{array}\right], \quad B=\left[\begin{array}{lll}
0 & 0 & 0 \\
0 & \Theta & 0 \\
0 & 0 & \Phi
\end{array}\right], \quad U=\left[\begin{array}{c}
\rho \\
u \\
T
\end{array}\right] .
$$


and where $\bar{u}$ is the mean normal velocity close to the solid wall, $a=\bar{c} / \sqrt{\gamma}, b=\bar{c} \sqrt{(\gamma-1) / \gamma}$ and $\bar{c}$ speed of sound. The coefficients $\Theta, \Phi$ are of order one and Eq. (7) is the symmetrized version of the one-dimensional Navier-Stokes equations ${ }^{12}$.

The dependent variables $\rho, u, T$ are scaled versions of the density, the normal velocity and the temperature, respectively. It can be shown ${ }^{13}$ (using the energy-method) that Eq. (7) is well-posed for $\bar{u} \leq 0$.

Similar to the scalar problem we discretize Eq. (7) using the SBP operators in Eq. (3) above and obtain the system

$$
V_{t}+\left(P^{-1} Q \otimes A\right) V=\varepsilon\left(P^{-1} M \otimes B\right) V+P^{-1}\left(E_{0} \otimes \Sigma\right) V
$$

where $\otimes$ is the so called Kronecker product ${ }^{4,14-16}$ and where the weak boundary conditions are contained in the last penalty term.

With the procedure above we now look at the spectra of the semi discrete equations including the boundary conditions and penalty terms which is obtained by looking at the eigenvalues of the matrix $C$ ( $C$ contains all the terms in Eq. (9))

$$
\frac{d}{d t} V=C V
$$

and where non-positive eigenvalues are required for stability. At the right boundary (at $\mathrm{x}=1)$ we specify all the variables $(\rho, u$ and $T)$ weakly using penalty terms. Figure 1 displays the spectra for the three viscous operators. All eigenvalues are negative for the selected parameters with penalty strength parameter $\sigma=1$ for all operators. The two compact operators have a very similar appearance but the eigenvalues with maximum real part differ slightly as can be seen from Table 1 below. The second compact operator seems to require a somewhat higher value of the penalty strength parameter than that derived for the scalar model problem and is unstable if $\sigma_{2} \leq 0.81$. Numerical calculations for the test cases below confirm that a higher penalty strength parameter is required for this operator. For the chosen set of parameters, the two other operators are stable for penalty strength parameters with values somewhat lower than those derived for the scalar model problem.

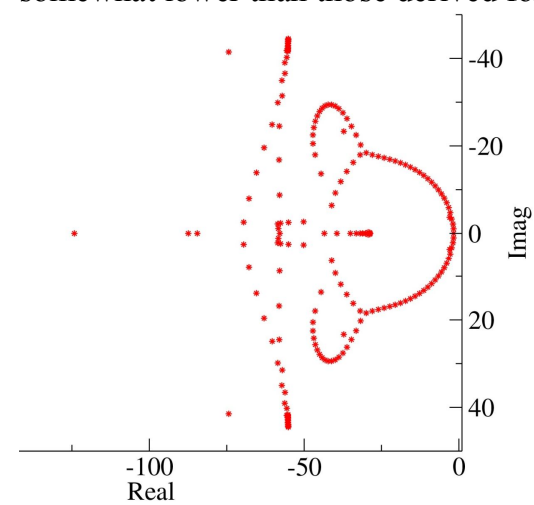

Figure 1. Spectra of $C$ for the linearized Navier-Stokes

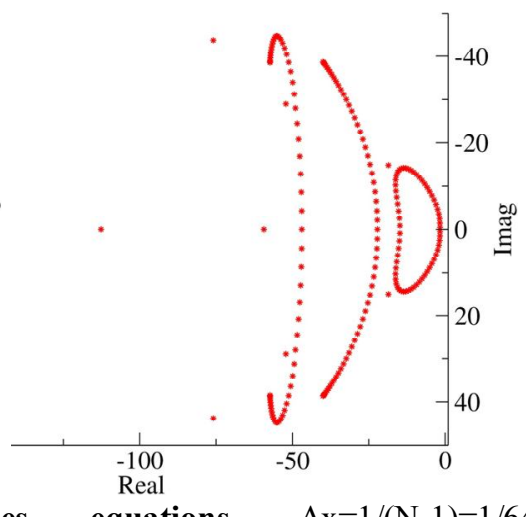
$\bar{u}=-0.8 ; \varepsilon=0.02 ; \sigma=1$. From left to right: Compact operator 1, Compact operator 2, Wide operator.

Table 1. Maximum real eigenvalues $\lambda$ of $C$ in Eq. (10), $\bar{u}=-0.8 ; \varepsilon=0.02$.

\begin{tabular}{llll}
\hline \hline$\sigma$ & Coarse op. 1 & Coarse op. 2 & Wide op. 1 \\
& Real $\left(\lambda_{\text {max }}\right)$ & $\operatorname{Real}\left(\lambda_{\max }\right)$ & $\operatorname{Real}\left(\lambda_{\max }\right)$ \\
\hline 0.75 & -1.69 & 0.60 & -1.58 \\
1 & -1.69 & -1.32 & -1.68 \\
2 & -1.71 & -1.65 & -1.71 \\
\hline \hline
\end{tabular}




\section{The flow solver Edge}

The CFD solver employed in the calculations is the Edge code which is an edge- and node-based Navier-Stokes flow solver applicable for both structured and unstructured grids ${ }^{8-10}$. Edge is based on a finite volume formulation where a median dual grid forms the control volumes with the unknowns allocated in the centres. The governing equations are integrated with a line-implicit approach in areas with highly stretched elements and explicitly elsewhere with a multistage Runge-Kutta scheme to steady state and with acceleration by Full Approximation Storage (FAS) agglomeration multigrid. A large number of turbulence models are available. Throughout this paper, a central scheme is used for the convection to which a small amount of numerical dissipation is added. This applies for the mean flow equations and for turbulent

There are numerous boundary conditions in Edge for walls, external boundaries as well as periodic boundaries. All of these boundary conditions are implemented weakly. The different stencils for the viscous terms described above are implemented for the viscous terms of the governing mean flow equations and turbulent diffusive terms. The implementation is done under the assumption that the grid is structured normal to the surface which is usually the case for practical applications on stretched RANS grids, e.g. through prismatic layers. The penalty formulation is applied to the equations where data is supplied which include no-slip velocity, iso-thermal conditions and turbulent boundary data.

\section{The semi-discrete Navier-Stokes equations in an edge based flow solver}

Consider a control volume $V_{i}$ for an arbitrary node with subscript $i$. The spatial discretization of the NavierStokes equations with a finite volume formulation on an unstructured grid for this node may be written in semidiscrete form as

$$
V_{i} \frac{\partial q_{i}}{\partial t}+\sum_{k=1}^{N_{i}} f_{i k}+\widetilde{f}_{i}=\sum_{k=1}^{N_{i}} g_{i k}+\widetilde{g}_{i}
$$

where $q_{i}=\left(\rho_{i}, u_{i}, E_{i}\right)$ contains the conservative variables of density, velocity vector and total energy for node $i . f_{i k}$ denotes the convective flux between nodes $i$ and $k, N_{i}$ is the number of nodes connected with an edge to node $i, g_{i k}$ the corresponding viscous flux. The terms $\widetilde{f}_{i}, \widetilde{g}_{i}$ denote the convective and viscous boundary fluxes to node $i$, provided the node is located on a boundary, otherwise these quantities vanish. The convective and viscous fluxes may be formulated as

$$
f_{i k}=\left(\begin{array}{c}
\rho_{\mathrm{i} k}\left(u_{i k} \cdot S_{i k}\right) \\
\rho_{i k}\left(u_{i k} \cdot S_{i k}\right) u_{i k}+p_{i k} S_{i k} \\
\left(u_{i k} \cdot S_{i k}\right)\left(E_{i k}+p_{i k}\right)
\end{array}\right)+A D_{i k} ; \quad g_{i k}=\left(\begin{array}{c}
0 \\
\tau_{i k} S_{i k} \\
u_{i k} \tau_{i k} S_{i k}-\kappa \nabla T_{i k} S_{i k}
\end{array}\right)
$$

where $S_{i k}$ denotes control surface along the edge between the two nodes $i$ and $k, u_{i k}$ the average velocity $\left(u_{i k}=1 / 2\left(u_{i}+u_{k}\right)\right)$ on the edge between the nodes and $A D_{i k}$ numerical dissipation ${ }^{8} . \tau_{i k}$ is the stress tensor, $T_{i k}$ denotes the average temperature and $\kappa$ the thermal conductivity coefficient. The stress tensor is divided in two parts with a first part based on a thin-layer approximation ${ }^{17}$

$$
\tau_{i k} S_{i k}=\left(\tau_{i k} S_{i k}\right)_{t l}+\left(\tau_{i k} S_{i k}\right)_{t d}=\mu\left(\frac{\partial u}{\partial n}+\frac{1}{3}\left(\frac{\partial u}{\partial n} \cdot n_{i k}\right) n_{i k}\right)\left|S_{i k}\right|+\left(\tau_{i k} S_{i k}\right)_{t d}
$$

where $S_{i k}=n_{i k}\left|S_{i k}\right|, \mu$ the dynamic viscosity or the sum of dynamic and turbulent viscosity for turbulent flow. The remaining tangential derivatives $\left(\tau_{i k} S_{i k}\right)_{t d}$ that are added from nodal Green-Gauss integration,

$$
\nabla \varphi_{i} \approx \frac{1}{V_{i}}\left(\sum_{k=1}^{n_{i}} \varphi_{i k} S_{i k}+\varphi_{i} S_{i}\right)
$$


to have a full viscous operator and where $\varphi_{i}, S_{i}$ are boundary quantities if the node $i$ is located on a boundary, otherwise these quantities vanish.

As indicated by Eq. (11), the computation of fluxes is done in two steps in the code. In the first step the fluxes are computed in a loop over all edges in the entire computational domain, the fluxes are added and subtracted to the corresponding residuals of the two nodes involved. In the second step, the boundary flux is computed and added to the residuals of the boundary nodes to close the control volume.

Our focus is on wall boundary conditions. On a wall boundary at node $i=0$ the convective boundary flux becomes, assuming a zero normal velocity component,

$$
\widetilde{f}_{0}=\left(\begin{array}{c}
0 \\
p_{0} S_{0} \\
0
\end{array}\right)
$$

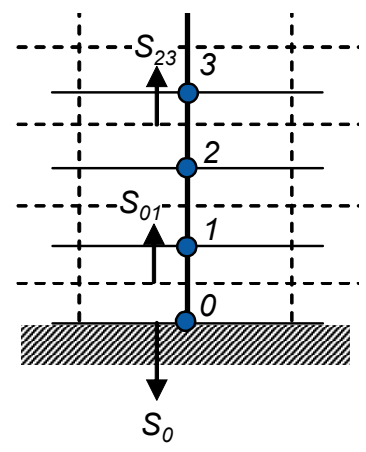

Figure 2. Wall boundary grid (solid line) with its dual grid (dashed).

The convention from Fig. 2 is used. The boundary surface vector $S_{0}$ is directed out of the computational domain. Below we demonstrate how the viscous wall flux $\widetilde{g}_{i}$ is computed corresponding to the different viscous discretizations for the scalar model equation above. The discretizations vary for the thin-layer part of the stress tensor. The viscous wall flux may be formulated as

$$
\tilde{g}_{0}=\left(\begin{array}{c}
0 \\
\left(\tau_{0} S_{0}\right)_{t l} \\
0
\end{array}\right) ; \quad\left(\tau_{0} S_{0}\right)_{t l}=\mu\left(\frac{\partial u}{\partial n}+\frac{1}{3}\left(\frac{\partial u}{\partial n} \cdot n_{0}\right) n_{0}\right)\left|S_{0}\right|
$$

where the tangential derivatives for the momentum equation from Eq. (13) are left out and assumed not to contribute to the boundary flux. In the description below the weak no-slip wall boundary conditions assume adiabatic conditions for the temperature, i.e. zero total energy wall flux. The weak conditions can be applied to other quantities with known values such as isothermal wall temperature and turbulent wall quantities.

1. Implementation of compact viscous operator 1

The normal derivative in Eq. (13) is approximated in a compact way ${ }^{18}$ as

$$
\frac{\partial u}{\partial n_{i k}}=\frac{u_{k}-u_{i}}{\left|x_{k}-x_{i}\right|}
$$

where $x_{i}, x_{k}$ are the coordinates of the two nodes. The discretization can be shown to lead to a positive and stable discretization of the Laplace operator ${ }^{19}$ but not necessarily to a consistent approximation on all types of grids.

The derivation of the wall flux follows the approach in Eliasson et al. ${ }^{6}$ in which the boundary normal derivatives are computed as:

$$
\frac{\partial u}{\partial n}=-\frac{\left(u_{1}-u_{0}\right)}{\left|x_{1}-x_{0}\right|}+\sigma \frac{\left(U-u_{0}\right)}{\left|x_{1}-x_{0}\right|}
$$

and inserted in the wall boundary flux in Eq. (16). Indices 0 and 1 denote the boundary node and the first interior node respectively according to Fig. 2 showing the notation of nodes with the primary (solid) and dual grid (dashed) at a wall boundary and where $S_{0}=n_{0}\left|S_{0}\right|$. Note that the first term in the expression for $\partial u / \partial n$ is a term to cancel out the contribution from the interior loop over the edges. $\sigma$ is the penalty strength parameter defined above and $\sigma \geq 1 / 4$ is required for stability as derived for the scalar model equation. Note that it is assumed that the grid is structured normal to the wall which is typical for hybrid unstructured grids with quadrilateral (2D) or prismatic 
(possibly hexahedral) near-wall elements (3D). $U$ in Eq. (18) denotes the prescribed wall velocity, typically $U=0$ on a no-slip wall, but it is included here to simplify the explanation. The expression for $\partial u / \partial n$ is then inserted in the boundary wall flux $\widetilde{g}_{0}$ in Eq. (16).

\section{Implementation of compact viscous operator 2}

The second compact operator has the same interior fluxes as the first operator with the normal derivative approximated as in Eq. (17). The boundary momentum flux is different though. To obtain the stencil for the model problem in Eq. (5), the velocity gradient in the first interior node is used:

$$
\frac{\partial u}{\partial n}=-2 \frac{\left(u_{1}-u_{0}\right)}{\left|x_{1}-x_{0}\right|}-\nabla u_{1} \cdot n_{0}+\sigma \frac{\left(U-u_{0}\right)}{\left|x_{1}-x_{0}\right|}
$$

which is inserted in the wall flux given in Eq. (16) and where $\nabla u_{1}$ is computed as in Eq. (14). Note that on an equidistant and orthogonal mesh $\nabla u_{1} \cdot n_{0}=-\left(u_{2}-u_{0}\right) /\left|x_{2}-x_{0}\right| \cdot \sigma \geq 5 / 8$ is required for stability.

\section{Implementation of wide viscous operator}

The wide operator has a different discretization in the interior as well as on the boundary. All fluxes are computed from averaged gradients in the nodes. The normal derivative is hence computed as

$$
\frac{\partial u}{\partial n_{i k}}=\frac{1}{2}\left(\nabla u_{i}+\nabla u_{k}\right) \cdot n_{i k} .
$$

The normal derivative at the boundary is also computed from the gradient on the boundary similar to the approach for the compact viscous operator 1 :

$$
\frac{\partial u}{\partial n}=\nabla u_{0} \cdot n_{0}+\sigma \frac{\left(U-u_{0}\right)}{\left|x_{1}-x_{0}\right|}
$$

where the first term on the right hand side is identical to the first term in Eq. (18) on an equidistant and orthogonal grid. $\sigma \geq 1 / 2$ is required for stability.

\section{Numerical results}

Numerical results are presented for steady state viscous calculations. The accuracy of the results is investigated by grid convergence studies on sequences of grids with the same topology and uniformly refined. All test cases employ no-slip adiabatic wall conditions and characteristic far-field boundary conditions. The viscous penalty strength parameters applied in the calculations are $\sigma_{1}=\sigma_{3}=1$ and a higher value $\sigma_{2}=2$ for the $2^{\text {nd }}$ compact viscous operator to obtain stability.

\section{Laminar flow over an airfoil}

The first test case is the laminar flow over an airfoil, NACA0012, at subsonic flow conditions and at a low Reynolds number; $M_{\infty}=0.5, \alpha=0^{\circ}$, $\mathrm{Re}=5000$. A sequence of 6 structured $\mathrm{O}$-grids are used for the grid convergence study, the coarser grids have been obtained by consecutively removing every second node from the finest grid in the two grid directions.

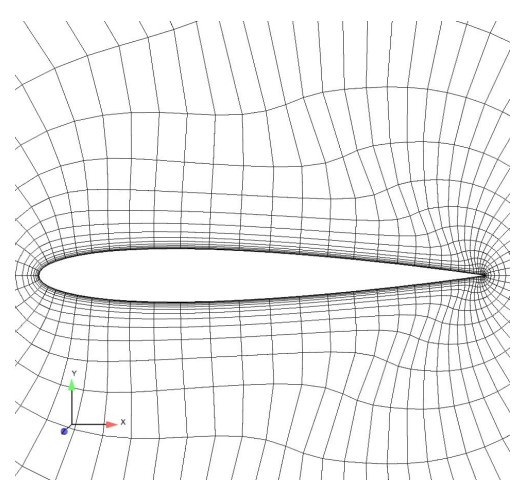

Figure 3. Coarsest NACA0012 Ogrid, $73 \times 29$ nodes. The finest grid contains about $2 \times 10^{6}$ nodes ( 2305 nodes around the airfoil, 897 nodes in normal direction), the coarsest grid about 2,000 grid nodes being displayed in Fig. 3 . The grids are refined at the leading and trailing edges and refined normal to the airfoil. The finest grid has a distance of $5 \times 10^{-5}$ 
chords between the wall and first interior nodes which corresponds to a cell Reynolds number of $\operatorname{Re}_{C}=0.25$ which should resolve viscous phenomena well.

The differences in the steady state convergence rate due to the three operators are negligible for this test case. The convergence of the drag and the pressure and friction drag components are given in Fig. 4. All viscous operators have a tendency to converge to the same drag, the difference on the finest grid is only about $0.04 \%$ or 0.2 drag cts (= $\left.0.6 \times 10^{-4}\right)$. The patterns of the differences are similar for the pressure and friction parts of the drag. The influence of the new boundary treatment of the $2^{\text {nd }}$ compact operator does not have the desired effect for this test case since the differences between the coarsest and finest grids increase compared to the $1^{\text {st }}$ compact operator.

The error of the drag with components are shown in Fig. 5 in which the error is computed as the difference between the drag values and the corresponding drag values on the finest grid. The errors for the three operators are similar and of about the same order of magnitude. The decay of the errors approach the expected $2^{\text {nd }}$ order decay as the grid is refined.
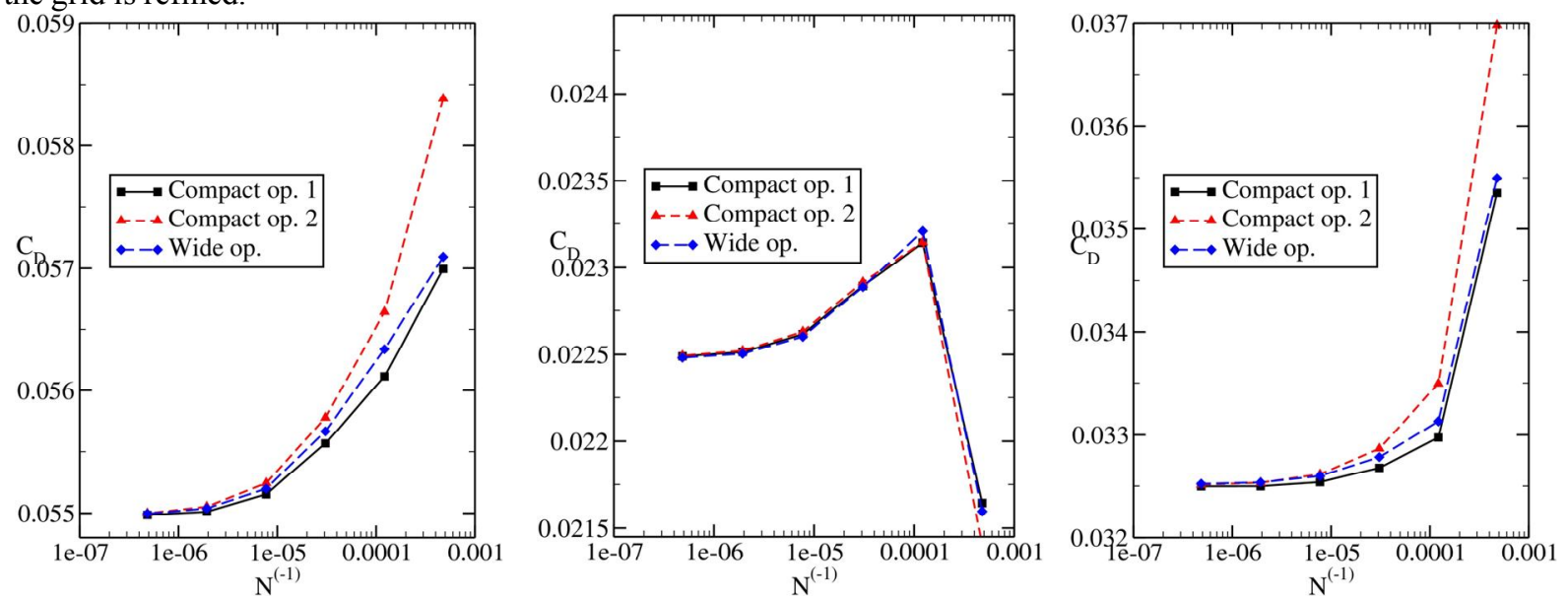

Figure 4. Convergence of drag as the grid is refined over NACA0012. Left: total drag. Mid: pressure drag. Right: friction drag. $\mathbf{N}$ denotes number of nodes. $M_{\infty}=0.5, \alpha=0^{\circ}, R e=5000$.
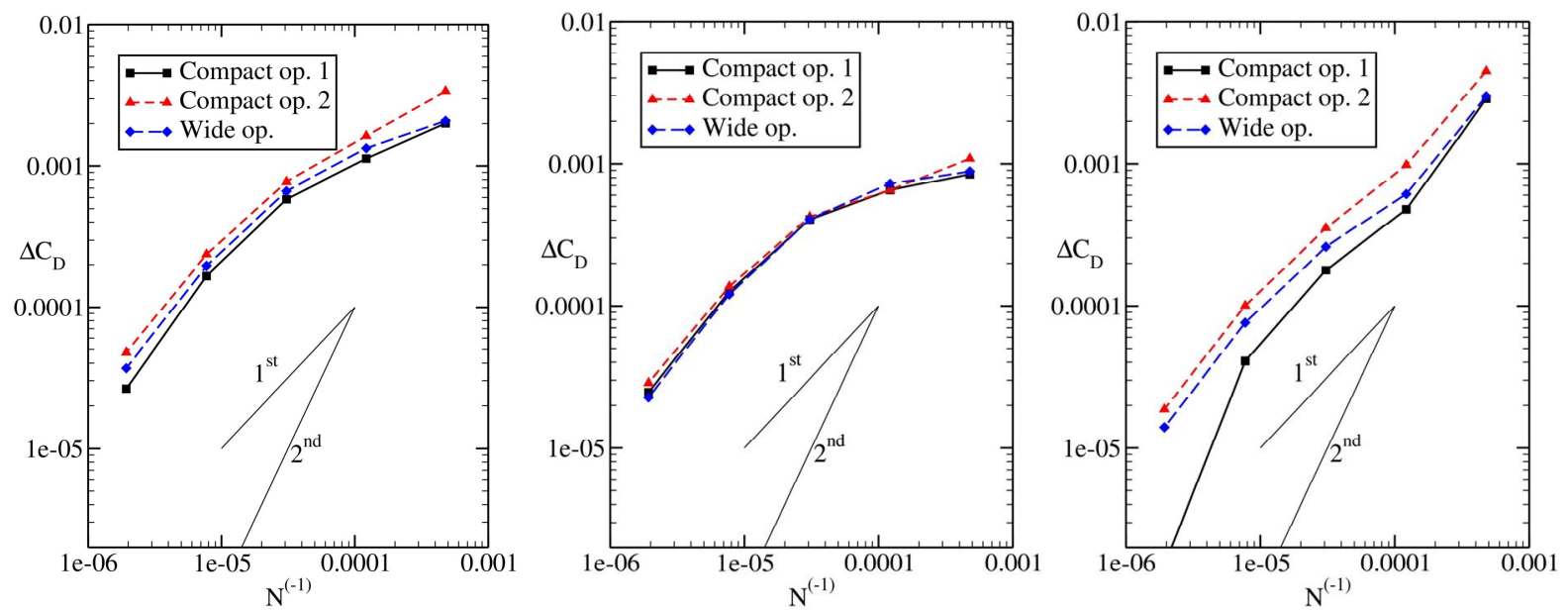

Figure 5. Error of drag as the grid is refined over NACA0012. Left: error for total drag. Mid: error for pressure drag. Right: error for friction drag. $\mathbf{N}$ denotes number of nodes. $M_{\infty}=0.5, \alpha=0^{\circ}, \operatorname{Re}=500$.

\section{E. Laminar flow over an analytical 3d body of revolution}

The second test case is the three-dimensional flow over a $3 \mathrm{~d}$ body of revolution. The geometry is a streamlined body based on a $10 \%$ thick airfoil with boundaries constructed by a surface of revolution. This test case is one of the test cases for the international workshop on higher order CFD methods for which numerous numerical results are available, see http://www.dlr.de/as/desktopdefault.aspx/tabid-8170/13999_read-35550/ for details. The laminar viscous test case is considered; $M_{\infty}=0.5, \alpha=1^{\circ}, \mathrm{Re}=5000$. Three supplied structured grids from the workshop were used. The three successively coarsened grids, for the entire configuration, contain $6.3 \times 10^{6}, 0.81 \times 10^{6}, 100 \times 10^{3}$ nodes 
and about the same number of hexahedral elements. In addition to the supplied grids, an extra fine grid (denoted Xfine) was generated from the fine grid by doubling nodes in all directions, this grid contains $51 \times 10^{6}$ nodes. The finest grid has a distance of $7 \times 10^{-4}$ characteristic length scales between the wall and first interior nodes which corresponds to a cell Reynolds number of $\operatorname{Re}_{C}=3$ which should be sufficient to resolve viscous phenomena reasonably well. The surface grid and a cut at the symmetry plane $(\mathrm{y}=0)$ are displayed in Fig. 6.

Figure 7 shows the computed lift, drag and viscous drag components for the three viscous operators as the grid is refined. There are rather small variations in the computed lift and reasonably good agreement with other numerical results for which the predicted reference lift is $\mathrm{C}_{\mathrm{L}}{ }^{\text {ref }}=0.002577$. The best agreement with this value is obtained by the calculations using the wide viscous operator.
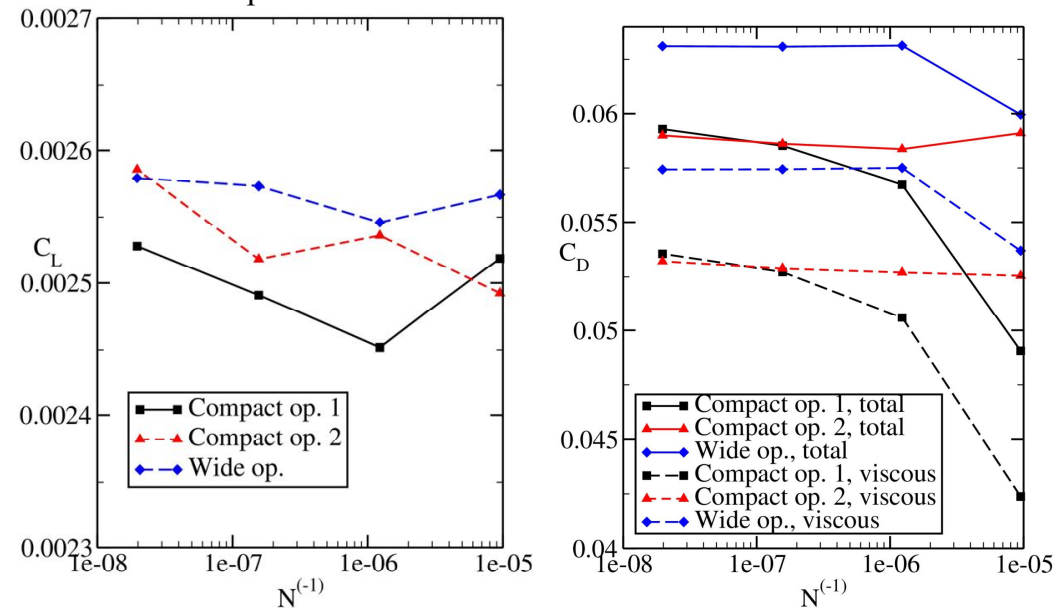

Figure 7. Computed forces for the 3d body of revolution. Left: lift. Right: total and viscous drag components. $\mathbf{N}$ denotes number of nodes. $M_{\infty}=0.5, \alpha=1^{\circ}, R e=5000$.

Cf

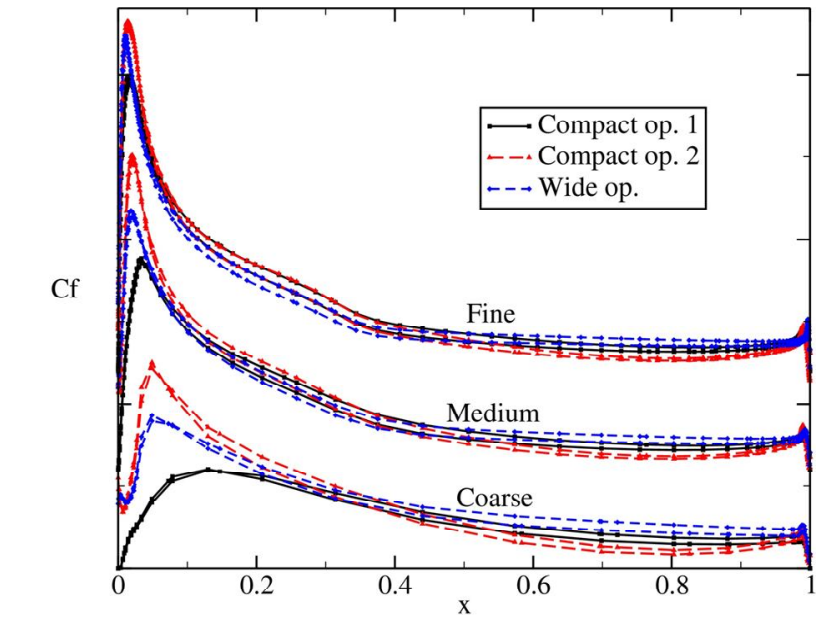

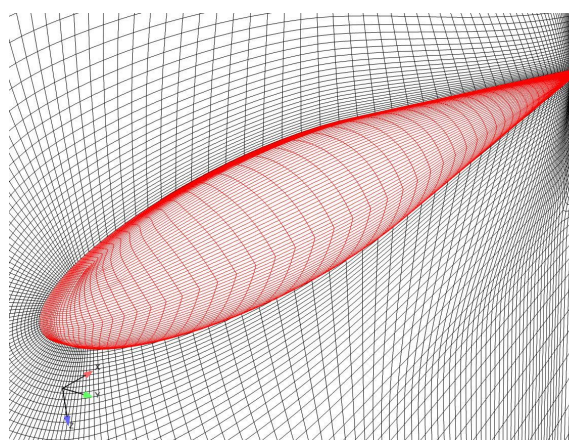

Figure 6. 3d body coarse surface mesh and cut in symmetry plane.

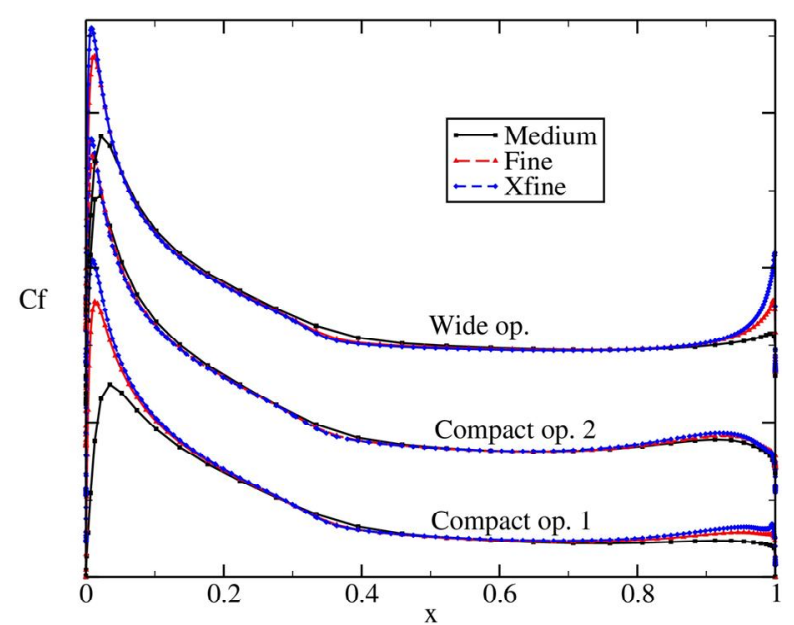

Figure 8. Stream-wise skin friction distribution on the $3 \mathrm{~d}$ body of revolution on three grids and viscous operators. Left: symmetry plane (y=0). Right: waist of body $(\mathbf{z}=\mathbf{0}) \cdot M_{\infty}=0.5, \alpha=1^{\circ}, R e=5000$.

There are larger differences between the computed results for the drag though. Calculations with the wide operator give substantially higher drag values than obtained with the compact discretizations, the variation on the three finest grids is also smallest with the wide operator. The drag comes mainly from the viscous operators; the pressure drag is fairly constant and substantially smaller. The reference drag value computed by the workshop participants is $C_{D}{ }^{\text {ref }}=0.0632$ which agrees very well with the drag obtained by the wide viscous operator, $C_{D}=0.0631$. The compact operators predict drag values that are about $7 \%$ or 45 drag cts lower. In spite of the lower predicted 
drag with the compact viscous operator, the new boundary condition with the $2^{\text {nd }}$ operator does increase the drag on coarser grids and hence reduces the grid dependency. The comparatively large difference in drag from the compact and wide viscous operators is surprising and needs to be explained. One possible explanation is that compact normal derivative in Eq. (17) does not lead to a consistent discretization of the Laplace operator ${ }^{19}$.

The stream-wise skin friction distributions in Fig. 8 at two cuts along the symmetry and body waist reveal the differences in the drag; the distributions are separated along the y-axis to fit in the same plot. There are some differences between the viscous operators that do not vanish as the grid is refined. The skin friction for each operator converge well though. The main differences between the operators occur at the downstream part of the body where the wide operator results in higher skin friction levels which is the main reason behind the higher integrated drag. The behavior at the trailing edge is also different between the three operators.

\section{F. Turbulent flow over an airfoil}

The third test case is the turbulent flow over an airfoil, NACA0012, at transonic flow conditions with a boundary layer shock interaction; $M_{\infty}=0.754, \alpha=2.57^{\circ}, \mathrm{Re}=20 \times 10^{6}$. Three unstructured hybrid grids are used with the same stream-wise resolution but with different wall normal distributions and distance between the wall and first interior node. The distances are $10^{-5}, 10^{-6}, 10^{-7}$ chords for the three grids.

One of the grids is displayed in Fig. 9. The grids are structured close to the airfoil with, in average, 30, 40, and 50 quadrilateral layers of cells and have 315 nodes around the airfoil. The grids contain a total number of $50 \times 10^{3}, 54 \times 10^{3}$,

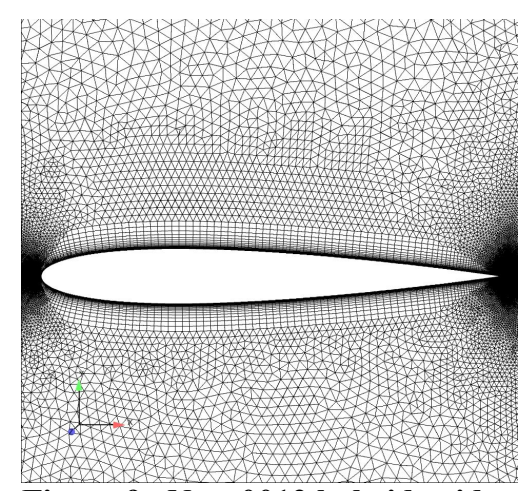

Figure 9. Naca0012 hybrid grid. $57 \times 10^{3}$ nodes, respectively, with triangular elements outside the quadrilaterals.

The Spalart-Allmaras turbulence model ${ }^{23}$ is used to model the turbulent flow. The high Reynolds number results in $\mathrm{y}^{+}$-values in the order of about $10,1,0.1$, respectively, for the three grids with increasing near wall resolution. The coarsest grid is then expected to be too coarse; the two finer grids should have a sufficient resolution.

A line-implicit integration approach is used to speed up the rate of convergence ${ }^{10}$. rates are obtained for the three viscous operators as displayed in Fig. 10.
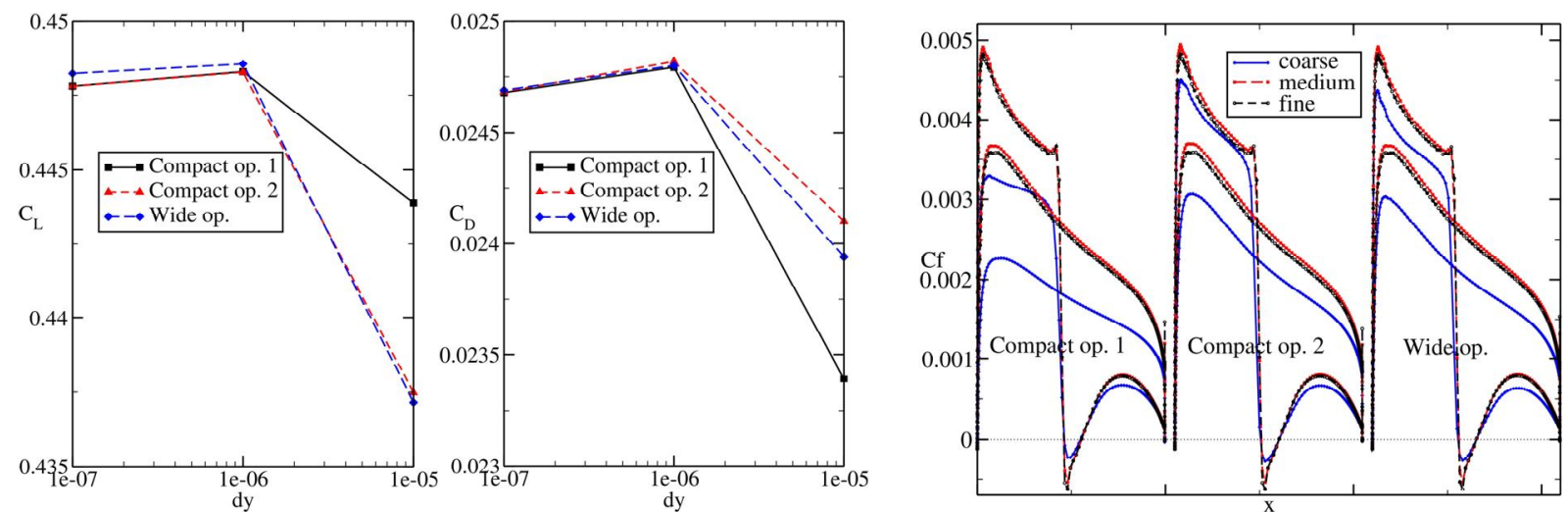

Figure 11. Lift (left), drag (mid) and skin friction as function of near wall resolution for the turbulent flow over NACA0012 with three viscous operators. $M_{\infty}=0.754, \alpha=2.57^{\circ}, R e=20 \times 10^{6}$.

Figure 11 displays the convergence of lift and drag as the mesh is refined as well as the skin-friction distributions for the three grids and viscous operators. There are very small differences in the integrated lift, the $1^{\text {st }}$ compact operator deviate somewhat on the coarsest grid from the other operators on the same grid. The coarse grid drag is improved by the $2^{\text {nd }}$ compact viscous operator which is also visible in the skin friction. In general, there are very small differences between the solutions on the two finer grids. 


\section{G. Turbulent flow over the DPW4 CRM configuration}

The last test case is a larger test from the $4^{\text {th }}$ drag prediction workshop, DPW4 ${ }^{20-22}$. The configuration is a common wing-body-tail configuration and is denoted the Common Research Model (CRM).

The flow conditions are $M_{\infty}=0.85, \mathrm{Re}=5 \times 10^{6}$ and the calculations in this investigation are made according to the instructions for the grid convergence studies at a constant lift force $\mathrm{C}_{\mathrm{L}}=0.5$ and $0^{\circ}$ tail incidence; the angle of attack is adjusted to obtain the requested lift. Three unstructured hybrid grids are used that were supplied by DLR and used earlier ${ }^{20}$. The grids contain unstructured quadrilateral surface elements

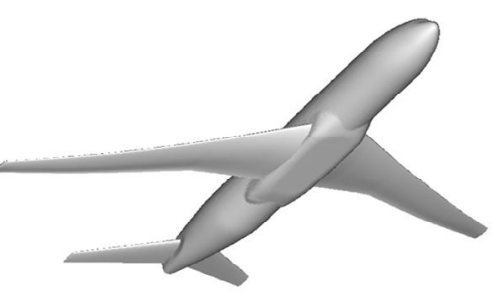

Figure 12. DPW4 CRM configuration.

with a few surface triangles. The surface grids are expanded normal to the wall resulting in hexahedral and prismatic elements. Outside of this region there are tetrahedral elements to the far-field boundary. The grids satisfy $\mathrm{y}^{+} \leq 1$ on all grids and have approximately a uniform refinement. The grids contain about $4 \times 10^{6}, 11 \times 10^{6}$ and $34 \times 10^{6}$ nodes.

The Spalart-Allmaras turbulence model is used to model the turbulent flow ${ }^{23}$. A line-implicit integration approach is used to speed up the rate of convergence ${ }^{10}$. The convergence of the density residual and lift coefficient for the three viscous operators is displayed in Fig. 13 for the medium grid size. The rate of convergence is very similar for the lift for all three operators. The density residual with wide operator does not converge as well though as for the compact operators. This may be due to the lack of damping from the viscous terms of high frequency errors and thus would require a somewhat higher level of numerical dissipation.

The convergence of integrated drag, drag components and

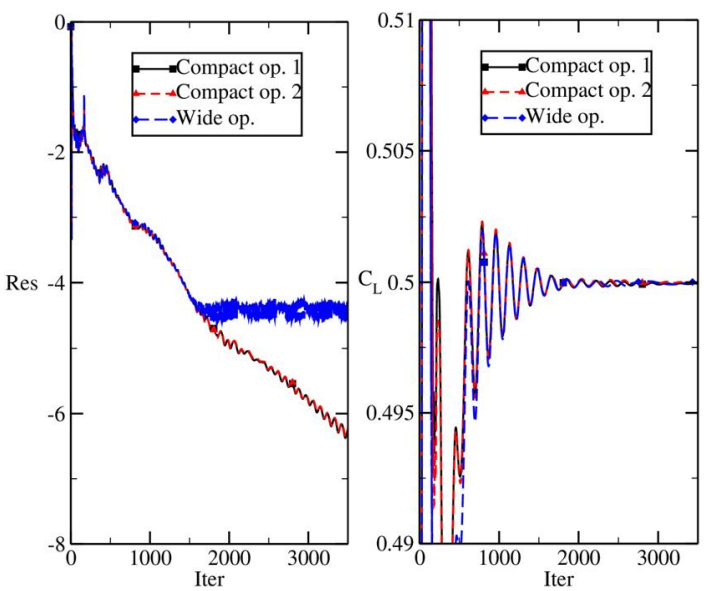

Figure 13. Steady state convergence rate of density residual and $C_{L}$. DPW4 medium grid. pitching moment are displayed in Fig. 14. The quantities are plotted as function of $N^{-2 / 3} \sim h^{2}$ which, ideally, should give convergence with straight lines assuming a second order accurate scheme and uniform refinement. The convergence is fairly good with all three operators and the differences in the results from the viscous operators are reduced as the grid is refined. The $2^{\text {nd }}$ compact operator reduces the mesh dependency slightly. The $1^{\text {st }}$ operator has a difference of $4.5 \mathrm{drag}$ cts between the coarse and fine grids whereas the $2^{\text {nd }}$ operator has a difference of $4.1 \mathrm{cts}$. The smallest difference is obtained with the wide operator though giving only 3.4 drag cts difference.
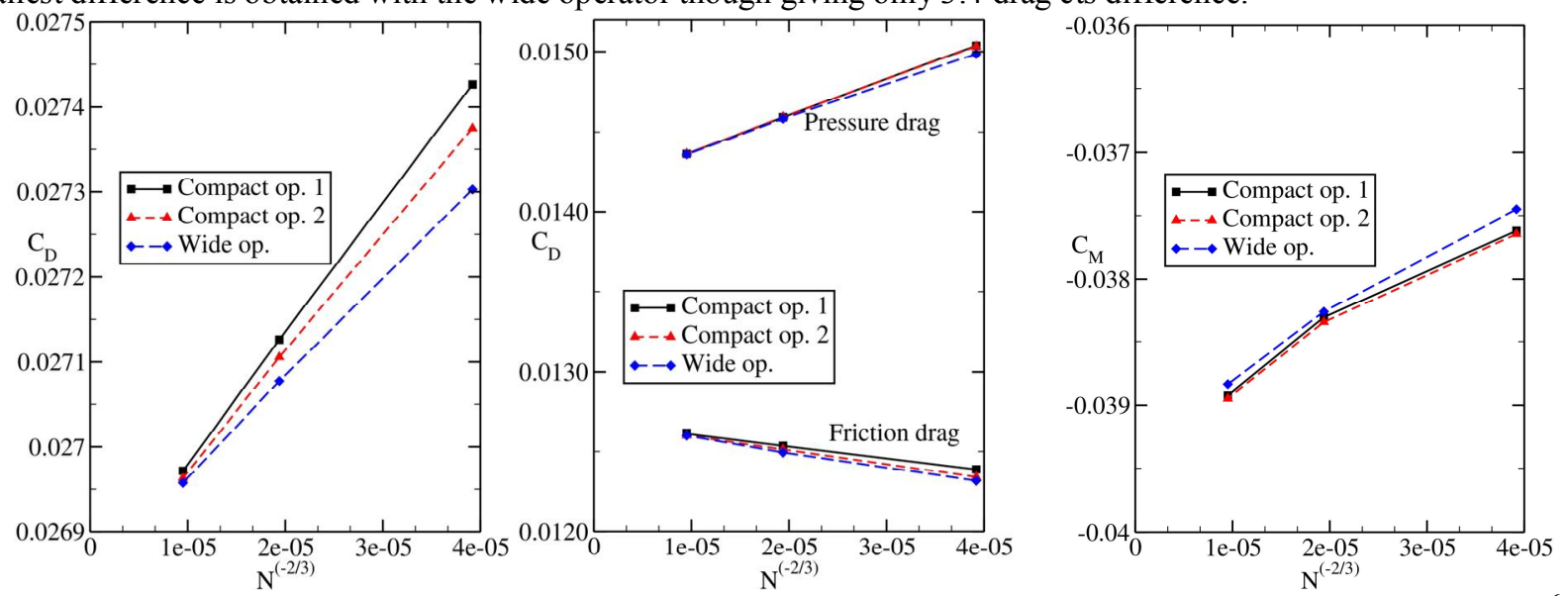

Figure 14. Convergence of drag and moment, $\mathbf{N}$ is the number of nodes, DPW4. $M_{\infty}=0.85, R e=5 \times 10^{6}$,

$C_{L}=0.5$. Left: Total drag. Middle: Drag decomposed into pressure and friction parts. Right: Pitching moment.

Apart from the differences in integrated forces and moments only small differences are observed in the converged solutions. The skin friction patterns reveal the flow is attached on the entire upper side of the wing, the main difference is observed inboard close to the wing-body junction as can be seen in Fig. 15 for two span-wise 
sectional cuts where the cut at $11.5 \%$ span is located in this region. The reason for the deviations is most likely due to a relatively coarse surface grid resolution in this region.

\section{Discussion}

An investigation of three different discrete numerical discretizations of the viscous terms in the Navier-Stokes equations is presented. The investigation is carried out for an edge-based solver for unstructured grids. Two of the discretizations have a compact discretization of the normal derivatives (including the Laplace and thin-layer part of the viscous terms) with different no-slip wall boundary conditions. The third discretization involves a wide operator which is obtained when nodal gradient only are used to compute the viscous fluxes. The boundary conditions are

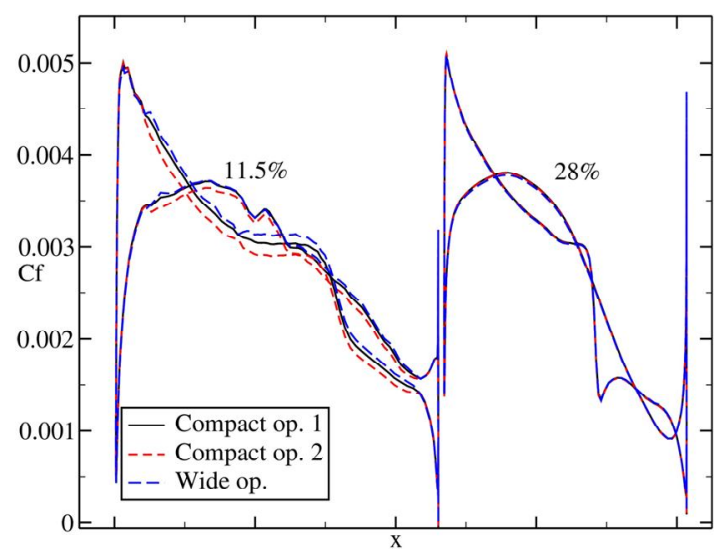

Figure 15. DPW4 skin friction distributions at $11.5 \%$ and $28 \%$ span. Medium grid.

implemented in a weak sense with penalty type boundary conditions. A theoretical investigation is carried out for a scalar model problem and limits are given to the penalty strength parameters to ensure stable discretizations. The implementation of the operators including boundary treatment is described as well.

The viscous operators are analyzed for several steady state test cases where grid convergence is studied on sequences of successively refined grids. For most test cases there are relatively small differences between the computed results. The $2^{\text {nd }}$ compact discretization is locally more accurate at the boundary compared to the $1^{\text {st }}$ discretization and is introduced with the intention to decrease mesh dependency and to increase accuracy on coarser grids. For three out of four test cases the mesh dependency is actually decreased, for the first and simplest test case the situation is reversed though. Although the wide viscous operator has larger truncation errors than the other schemes, it performs surprisingly well for all test cases and provides as accurate results as obtained with the compact schemes. For several test cases this scheme gives the smallest variation between the coarsest and finest grids.

For three out of four test cases the three viscous operators seem to converge towards the same solutions as the grids are refined. One exception is the three-dimensional laminar flow over a bluff body where the coarse operator converges to a drag that is approximately $7 \%$ higher than that obtained with the compact operators. The higher drag is in line with other numerical results and is therefore believed to be the most accurate result. The reason for the under-prediction with the two compact schemes is not known and is a subject for future studies.

The steady state convergence rate is in general not much affected by the viscous terms; very similar convergence rates are obtained. One exception is the large three-dimensional turbulent drag prediction test case where the residuals "hang" after a while using the wide operator whereas with the compact operators the residuals continue to converge. One possible explanation is that the wide operator does not damp the highest frequencies as the compact operators do and may hence need a bit more numerical dissipation. Despite the somewhat high level of residuals the convergence rate of the integrated forces is about the same for all the schemes.

\section{Appendix}

We present the specific forms of the stencils that satisfy the SBP stability requirements being second order accurate. We consider the discrete form of Eq. (1) as defined by Eq. (2) where $v$ is defined as the error of the discrete solution. The first derivative is approximated as:

$$
u_{x} \approx P^{-1} Q v, \quad P=\Delta x\left[\begin{array}{cccc}
1 / 2 & & & \\
& 1 & & \\
& & 1 & \\
& & & \ddots
\end{array}\right], Q=\frac{1}{2}\left[\begin{array}{cccc}
-1 & 1 & & \\
-1 & 0 & 1 & \\
& -1 & 0 & 1 \\
& & & \ddots
\end{array}\right], P^{-1} Q=\frac{1}{2 \Delta x}\left[\begin{array}{cccc}
-2 & 2 & & \\
-1 & 0 & 1 & \\
& -1 & 0 & 1 \\
& & & \ddots
\end{array}\right], v=\left[\begin{array}{c}
v_{0} \\
v_{1} \\
v_{2} \\
\vdots
\end{array}\right]
$$

where the lower part of the matrices are omitted. $Q$ is skew symmetric and $E_{0}$ has only one non-zero element, 


$$
Q+Q^{T}=\left[\begin{array}{cccc}
-1 & & & \\
& 0 & & \\
& & 0 & \\
& & & \ddots
\end{array}\right], E_{0}=\left[\begin{array}{llll}
1 & & & \\
& 0 & & \\
& & 0 & \\
& & & \ddots
\end{array}\right] .
$$

We define the following matrices:

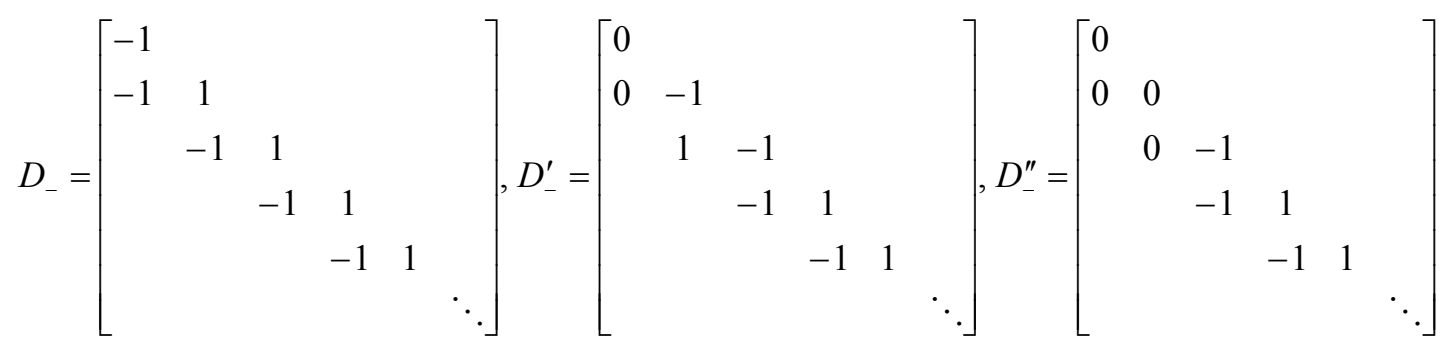

Following Eq. (2) and multiplying with $v^{T} P$ we derive the following equation for the decay of the solution:

$$
\frac{d}{d t}\|v\|_{P}^{2}=a(1-2 \tau) v^{T} E_{0} v+\varepsilon v^{T}\left(M+M^{T}\right) v-\varepsilon \frac{2 \sigma}{\Delta x} v^{T} E_{0} v
$$

where the norm is defined as $\|v\|_{P}=v^{T} P v$. Stability is obtained if the right hand side can be shown to be nonpositive thus preventing error growth in time. For convection only $(\varepsilon=0)$ it is obvious that $\tau \geq 1 / 2$ must be satisfied. Below we show the conditions for the two last terms on the right hand side in Eq. (22) to be non-positive for the different viscous operators defined in Eqs. (4)-(6) above, the convective condition corresponding to the first term on the right hand side in Eq. (22) is left out.

\section{The compact viscous operator 1}

The proof follows in principle the proof given by Eliasson et al. ${ }^{6}$. The viscous operator in Eq. (4) is written as

$$
M=-\frac{1}{\Delta x} D_{-}^{\prime} D_{-}^{\prime T}+\frac{1}{\Delta x}\left[\begin{array}{cccc}
0 & 0 & & \\
1 & -1 & & \\
& & 0 & \\
& & & \ddots
\end{array}\right]
$$

leading to the following expression for the decay of solution:

$$
\begin{aligned}
& \frac{d}{d t}\|v\|_{P}^{2}=-\frac{2 \varepsilon}{\Delta x} v^{T} D_{-}^{\prime} D_{-}^{\prime T} v+\frac{\varepsilon}{\Delta x} v^{T}\left[\begin{array}{cccc}
-2 \sigma & 1 & & \\
1 & -2 & & \\
& & 0 & \\
& & & \ddots
\end{array}\right] v= \\
& -\frac{2 \varepsilon}{\Delta x} \sum_{i=1}\left(v_{i+1}-v_{i}\right)^{2}+\frac{\varepsilon}{\Delta x}\left[\begin{array}{l}
v_{0} \\
v_{1}
\end{array}\right]^{T}\left[\begin{array}{cc}
-2 \sigma & 1 \\
1 & -2
\end{array}\right]\left[\begin{array}{l}
v_{0} \\
v_{1}
\end{array}\right]
\end{aligned}
$$


The first term on the right hand side is obviously non-positive. Then the $2 \times 2$ matrix in the last term must have non-positive eigenvalues which is obtained if $\sigma=\sigma_{1} \geq 1 / 4$.

2. $\quad$ The compact viscous operator 2

The viscous operator in Eq. (5) can be written as

$$
M=-\frac{1}{\Delta x} D_{-}^{\prime \prime} D_{-}^{\prime \prime}+\frac{1}{\Delta x}\left[\begin{array}{ccccc}
1 / 2 & -1 & 1 / 2 & & \\
1 & -2 & 1 & & \\
& 1 & -1 & & \\
& & & 0 & \\
& & & & \ddots
\end{array}\right]
$$

leading to the following equation for the decay of error:

$$
\begin{aligned}
& \frac{d}{d t}\|v\|_{P}^{2}=-\frac{2 \varepsilon}{\Delta x} v^{T} D_{-}^{\prime \prime} D_{-}^{\prime \prime T} v+\frac{\varepsilon}{\Delta x} v^{T}\left[\begin{array}{cccc}
1-2 \sigma & 0 & 1 / 2 & \\
0 & -4 & 2 & \\
1 / 2 & 2 & -2 & \\
& & 0 & \\
-\frac{2 \varepsilon}{\Delta x} \sum_{i=2}\left(v_{i+1}-v_{i}\right)^{2}+\frac{\varepsilon}{\Delta x}\left[\begin{array}{c}
v_{0} \\
v_{1} \\
v_{2}
\end{array}\right]^{T}\left[\begin{array}{ccc}
1-2 \sigma & 0 & 1 / 2 \\
0 & -4 & 2 \\
1 / 2 & 2 & -2
\end{array}\right] v= \\
v_{1} \\
v_{2}
\end{array}\right] .
\end{aligned}
$$

where the $3 \times 3$ matrix can be shown to have non-positive eigenvalues if $\sigma=\sigma_{2} \geq 5 / 8$.

3. The wide viscous operator

The viscous operator in Eq. (6) can be written as

$$
M=-\frac{1}{\Delta x} Q P^{-1} Q
$$

for which the which the decay of the solution can be written as

$$
\begin{aligned}
& \frac{d}{d t}\|v\|_{P}^{2}=\frac{\varepsilon}{\Delta x} v^{T}\left(Q P^{-1} Q+Q^{T} P^{-1} Q^{T}\right) v-\frac{2 \varepsilon}{\Delta x} \sigma v^{T} E_{0} v= \\
& -\frac{2 \varepsilon}{\Delta x} v^{T} Q^{T} P^{-1} Q v-\frac{\varepsilon}{\Delta x} v^{T}\left(E_{0} P^{-1} Q+Q^{T} P^{-1} E_{0}+2 \sigma E_{0}\right) v
\end{aligned}
$$

which after some algebra can be written as

$$
\frac{d}{d t}\|v\|_{P}^{2}=-\frac{\varepsilon}{2 \Delta x} \sum_{i=1}\left(v_{i+2}-v_{i}\right)^{2}+\frac{\varepsilon}{\Delta x}\left[\begin{array}{c}
v_{1} \\
v_{1}-v_{0}
\end{array}\right]^{T}\left[\begin{array}{cc}
-2 \sigma & -1 \\
-1 & -1
\end{array}\right]\left[\begin{array}{c}
v_{1} \\
v_{1}-v_{0}
\end{array}\right]
$$

The first term is quadratic and non-positive, the matrix of the second term has non-positive eigenvalues provided $\sigma=\sigma_{3} \geq 1 / 2$. 


\section{Acknowledgments}

The work has been supported by the EU project IDIHOM under contract No. ACP0-GA-2010-265780.

\section{References}

${ }^{1}$ Svärd, M. and Nordström, J., "A Stable High-Order Finite Difference Scheme for the Compressible Navier-Stokes Equations: No-Slip Wall Boundary Conditions”, Journal of Computational Physics, Vol. 227, 2008, pp. 4805-4824.

${ }^{2}$ Svärd, M., Carpenter, M. and Nordström, J., "A Stable High Order Finite Difference Scheme for the Navier-Stokes Equations, far-field Boundary Conditions”, Journal of Computational Physics, Vol. 225 (1), 2007, pp. 1020-1038.

${ }^{3}$ Nordström, J., Gong, J., van der Weide, E. and Svärd, M., "A stable and conservative high order multi-block for the compressible Navier-Stokes equations”, Journal of Computational Physics, Vol. 228, 2009, pp. 9020-9035.

${ }^{4}$ Carpenter, M., H., Nordström, J. and Gottlieb, D. "A Stable and Conservative Interface Treatment of Arbitrary Spatial Accuracy", Journal of Computational Physics, Vol 148 No. 2, 1999, pp. 341-365.

${ }^{5}$ Berg, J., Nordström, J. "Stable Robin Solid Wall Boundary Conditions for the Navier-Stokes Equations", Journal of Computational Physics, Vol 230, 2011, pp. 7519-7532.

${ }^{6}$ Eliasson, P., Eriksson, S., Nordström, J. "The Influence of Weak and Strong Wall Boundary Conditions on the Convergence to Steady-State of the Navier-Stokes Equations", AIAA-2009-3551.

${ }^{7}$ Nordström, J., Eriksson, S. and Eliasson, P., "Weak and Strong boundary procedures and convergence to steady-state of the Navier-Stokes equations", Journal of Computational Physics, Vol. 231, 2012, pp. 4867-4884.

${ }^{8}$ Eliasson, P., "EDGE, a Navier-Stokes Solver for Unstructured Grids", Proceedings to Finite Volumes for Complex Applications III, 2002, pp. 527-534.

${ }^{9}$ Eliasson, P., Weinerfelt, P., "Recent Applications of the Flow Solver Edge", Proceedings to 7th Asian CFD Conference, Bangalore, India, 2007.

${ }^{10}$ Eliasson, P., Weinerfelt, P., Nordström, J. "Application of a Line-implicit Scheme on Stretched Unstructured Grids", AIAA-2009-0163.

${ }^{11}$ Svärd, M., Nordström, J., "On the order of accuracy for difference approximations of initial-boundary value problems", Journal of Computational Physics, Vol. 218, 2006, pp. 333-352.

${ }^{12}$ Nordström, J. "The Influence of Open Boundary Conditions on the Convergence to Steady State of the Navier-Stokes Equation", Journal of Computational Physics, Vol. 85, No. 1, 1989, pp. 210-244.

${ }^{13}$ Nordström, J., Svärd, M. "Well-posed Boundary Conditions for the Navier-Stokes Equations", SIAM J. Num. Anal., Vol. 43, No. 3, 2005, pp. 1231-1255

${ }^{14}$ Nordström, J. and Carpenter, M. H. "Boundary and Interface Conditions for High Order Finite Difference Methods Applied to the Euler and Navier Stokes Equations", Journal of Computational Physics, Vol 148 No. 2, 1999, pp. 621-645.

${ }^{15}$ Nordström, J. and Carpenter, M. H. "High Order Finite Difference Methods, Multidimensional Linear Problems and Curvilinear Coordinates", Journal of Computational Physics, Vol 173, 2001, pp. 149-174.

${ }^{16}$ Nordström, J., Forsberg, K. Adamsson, C. and Eliasson, P. "Finite Volume Methods, Unstructured Meshes and Strict Stability", Applied Numerical Mathematics, Vol. 48, 2003, pp. 453-473.

${ }^{17}$ Gnoffo, P. A., "An Upwind-Biased, Point-Implicit Relaxation Algorithm for Viscous, Compressible Perfect Gas Flows", NASA TP-2953, 1990.

${ }^{18}$ Haselbacher, A., McGuirck, J. J., and Page, G.J., "Finite Volume Discretization Aspects for Viscous Flows on Mixed Unstructured Grids", AIAA Journal, Vol. 37, No. 2, 1999, pp. 177-184.

${ }^{19}$ Svärd, M. and Nordström, J. "Stability of finite volume approximations for the Laplacian operator on quadrilateral and triangular grids", Applied Numerical Mathematics, Vol. 51, 2004, pp. 101-125.

${ }^{20}$ Eliasson, P., Peng. S.-H., "Computations from the 4th Drag Prediction Workshop Using the Edge Solver", AIAA Paper $2010-4548$.

${ }^{21}$ Vassberg, J. C., Tinoco, E. N., Mani, M., Rider, B., Zickhur, T., Levy, D. Brodersen, O. P., Eisfeld, B., Crippa, S., Wahls, R. A., Morrison, J. H., Mavriplis, D. J. and Murayama, M. "Summary of the Fourth AIAA CFD Drag Prediction Workshop", 2010, AIAA 2010-4547.

${ }^{22}$ Morrison, J. H. "Statistical Analysis of CFD Solutions from the Fourth AIAA Drag Prediction Workshop", AIAA Paper 2010-4673, 2010.

${ }^{23}$ Spalart, P. R., and Allmaras, S. R., "A One-Equation Turbulence Model for Aerodynamic Flows", AIAA Paper 92-0439, 1992. 